\title{
p53 involvement in clonal hematopoiesis of indeterminate potential
}

\author{
Sisi Chen and Yan Liu
}

Department of Biochemistry and Molecular Biology, Department of Pediatrics, Herman B

Wells Center for Pediatric Research, Indiana University, Indianapolis, IN 46202, USA.

Correspondence to Yan Liu, PhD, Department of Pediatrics, Herman B Wells Center for

Pediatric Research, Indiana University, Indianapolis, IN 46202, USA. Tel:+1 317278 0333;

fax: 317274 8769; e-mail: liu219@iu.edu

This is the author's manuscript of the article published in final edited form as:

Chen, S., \& Liu, Y. (2019). P53 involvement in clonal hematopoiesis of indeterminate potential. Current Opinion in Hematology, 26(4), 235-240. https://doi.org/10.1097/MOH.0000000000000509 


\section{ABSTRACT}

\section{Purpose of review}

Clonal hematopoiesis of indeterminate potential (CHIP) increases with age and occurs when a single mutant hematopoietic stem cell (HSC) contributes to a significant clonal proportion of mature blood lineages. Somatic mutations in the TP53 gene, which encodes the tumor suppressor protein p53, rank in the top five among genes that were mutated in CHIP. This review focuses on mechanisms by which mutant p53 promotes CHIP progression and drives the pathogenesis of hematological malignancies, including myelodysplastic syndromes (MDS), and acute myeloid leukemia (AML).

\section{Recent findings}

TP53 was frequently mutated in individuals with CHIP. While clinical studies suggest that expansion of HSCs with TP53 mutations predisposes the elderly to hematological neoplasms, there is a significant gap in knowledge regarding the mechanisms by which TP53 mutations promote HSC expansion. Recent findings suggest that several cellular stressors, including hematopoietic transplantation, genotoxic stress, and inflammation, promote the expansion of HSCs with TP53 mutations. Further, TP53 mutations identified in CHIP cooperate with genetic and/or epigenetic changes in leukemogenesis.

\section{Summary}

TP53 mutations identified in CHIP are associated with increased risks of de novo and therapy-related hematological neoplasms. Thus, targeting mutant p53 and related pathways 
holds great potential in preventing CHIP progression and treating hematological malignancies.

\section{Keywords}

clonal hematopoiesis of indeterminate potential, age-related clonal hematopoiesis, TP53, p53, myelodysplastic syndromes, and acute myeloid leukemia

\section{KEY POINTS}

Tumor suppressor gene TP53 was frequently mutated in individuals with clonal hematopoiesis of indeterminate potential (CHIP).

Several cellular stressors, including transplantation, genotoxic stress, and inflammation, promote the expansion of hematopoietic stem cells (HSCs) with TP53 mutations.

TP53 mutations identified in CHIP cooperate with genetic and/or epigenetic changes in leukemogenesis.

Targeting mutant p53 and related pathways holds great potential in preventing CHIP progression and treating hematological malignancies. 


\section{INTRODUCTION}

Human aging is associated with an exponential increase in the occurrence of clonal hematopoiesis of indeterminate potential (CHIP) in aged individuals. CHIP occurs when a single mutant hematopoietic stem cell (HSC) contributes to a significant, measurable clonal proportion of mature blood lineages [1, 2m, 3]. CHIP is also known as age-related clonal hematopoiesis $(\mathrm{ARCH})[4 \bullet \mathbf{a}$. CHIP is associated with an increased risk of hematological malignancies, such as myelodysplastic syndromes (MDS) and acute myeloid leukemia (AML), as well as a higher incidence of other age-related pathological conditions such as cardiovascular disease (CVD) [5-10,11 ]. Given that CHIP is an age-dependent risk factor for MDS, AML, and CVD, preventing CHIP progression may prove to be beneficial for human health. However, mechanisms by which somatic mutations in blood cells contribute to the pathogenesis of age-related diseases are largely unknown. The vast majority of the mutations identified in CHIP are dispersed across the genome. However, five genes, including DNMT3A, TET2, ASXL1, JAK2 and TP53, have disproportionately high numbers of somatic mutations [5-7]. TP53 ranks in the top five among genes that were mutated in CHIP and the frequency of TP53 mutations increases with age $[5-9,12 m, 13 \boldsymbol{m}]$. TP53 gene encodes the tumor suppressor p53 protein [14]. This review will focus on the involvement of p53 in CHIP progression and pathogenesis of hematological malignancies, including MDS and AML.

\section{p53 in clonal hematopoiesis of indeterminate potential (CHIP)}

p53 is a transcription factor that regulates a large number of genes in response to a variety of cellular insults, including oncogene activation, DNA damage, and inflammation. These stressors activate p53 through post-translational modifications that result in augmented levels of p53 protein and transactivation activity [14-15]. Activated p53 induces growth arrest, 
apoptosis, DNA repair, and differentiation in damaged cells to suppress cellular transformation [14-15]. p53 is a critical regulator of hematopoietic stem cell behavior and we discovered that p53 maintains HSC quiescence and regulates DNA damage response [1617]. p53 activates the transcription of target genes to mediate DNA damage repair, growth arrest or apoptosis $[14,18]$. We have identified both Necdin and Gfi1 as p53 target genes in HSCs [16-17].

Like solid tumors, p53 function is always compromised in hematological malignancies, usually as a result of somatic mutations and deletions [14-15, 19-23]. TP53 ranks in the top five among genes that were mutated in CHIP (Fig. 1). The majority of TP53 mutations in CHIP are missense mutations. The remainder of the TP53 mutations are nonsense, frameshift, and splice site mutations $[5-7,12 \boldsymbol{- 1}, 13 \boldsymbol{-}]$. TP53 mutation spectrums in CHIP are similar to hematological malignancies, including MDS and AML [19-23]. While clinical studies suggest that expansion of HSCs with TP53 mutations predisposes the elderly to hematological neoplasms $[5-7,12 \boldsymbol{\bullet}, 13 \mathbf{m}]$, the mechanisms by which TP53 mutations promote HSC expansion during aging are largely unknown. We discovered that TP53 mutations identified in CHIP enhances the repopulating potential of HSCs, thereby conferring a competitive advantage to HSCs following transplantation stress [2400]. Hematopoietic transplantation also expanded p53 mutant clones in patients with hematological malignancies $[1300]$.

Modulation of gene transcription is an important mechanism for mutant p53 in cancer development [15]; however, how mutant p53 regulates gene expression in HSCs is largely unknown. Loss of epigenetic regulation of gene expression in aged HSCs contributes to aging phenotypes and dysregulated epigenetic control has been implicated in the 
pathogenesis of hematological malignancies [20-21, 25-26]. Indeed, recent studies revealed that some mutant p53 proteins increase the expression of epigenetic factors, including MLL1, MLL2, and MOZ, in human cancer cells [27]. However, we found that the expression of MII1, MII2, and Moz is comparable in p53 wild-type and mutant HSCs (S.C. and Y.L., unpublished data). As mutant p53 proteins have been shown to play context dependent roles in human cancer [15], it is possible that mutant p53 proteins may utilize different mechanisms to modulate gene expression in HSCs. We found that mutant p53 interacts with epigenetic regulator EZH2. EZH2, a key component of Polycomb repressive complex 2 (PRC2), catalyzes the trimethylation of lysine 27 of histone $\mathrm{H} 3(\mathrm{H} 3 \mathrm{~K} 27 \mathrm{me} 3)$ in cells [28]. Hematopoietic-specific deletion of Ezh2 impairs hematopoietic stem cell self-renewal and terminal differentiation [29]. We discovered that mutant p53 enhanced the association of $\mathrm{EZH} 2$ with the chromatin and increased the levels of $\mathrm{H} 3 \mathrm{~K} 27 \mathrm{~m} 3$ in genes regulating $\mathrm{HSC}$ selfrenewal and differentiation (S.C. and Y.L., unpublished data). Thus, we have uncovered a novel mechanism by which mutant p53 drives clonal hematopoiesis. Since common mutations identified in CHIP affect epigenetic modulators, including DNMT3A, TET2, and ASXL1 [5-7], our findings that mutant p53 modulates EZH2 activity and increases the levels of $\mathrm{H} 3 \mathrm{~K} 27 \mathrm{~m} 3$ in HSCs underscore the importance of dysregulated epigenetic control in CHIP development.

Most of TP53 mutations in human cancer lost their tumor suppressor function [15]. However, mounting evidence suggests that some mutant p53 proteins not only lose their 
tumor suppressor function, but also acquire new oncogenic properties that are independent of wild-type p53, known as gain-of-function (GOF) properties [15]. To ascertain the oncogenic effects of GOF TP53 mutations, $\mathrm{p} 53^{\mathrm{R} 248 \mathrm{~W}}$ and $\mathrm{p} 53^{\mathrm{R} 273 \mathrm{H}}$ mutant were engineered into the endogenous Trp53 locus in mice [30]. Homozygous $p 53^{R 248 W / R 428 W}$ and $p 53^{R 273 H / R 273 H}$ mice developed novel tumors compared to $p 53^{-/-}$mice [30]. Thus, GOF mutant $p 53$ proteins have enhanced oncogenic potential beyond the simple loss of $p 53$ function. Since EZH2 interacts with mutant p53 but not wild-type p53, our findings suggest that some TP53 mutations identified in CHIP may have GOF properties.

In transplantation assays, p53 mutant hematopoietic cells always outcompeted wildtype competitor cells and became clonal dominance [1300, 2400]. While we have identified a cell autonomous mechanism by which mutant p53 drives clonal hematopoiesis, recent studies indicate that mutations identified in CHIP may utilize cell non-autonomous mechanisms to promote clonal hematopoiesis [11•, 31ø]. For example, mutant hematopoietic cells displayed increased secretion of pro-inflammatory cytokines, including IL$1 \beta$ and IL-6 [11ø, 31øa]. We found that inhibition of inflammatory signaling in Tet2 mutant preleukemic cells mitigates clonal hematopoiesis [3100]. RNA-seq analysis revealed that inflammatory response genes are enriched in p53 mutant HSCs (S.C. and Y.L., unpublished data), suggesting that mutant p53 may drive clonal hematopoiesis through activating proinflammatory pathways. The potential cell non-autonomous mechanisms by which mutant p53 promotes HSC expansion during aging await future investigation.

\section{p53 in therapy-related CHIP}


TP53 mutations are commonly found in therapy related CHIP as well as in secondary

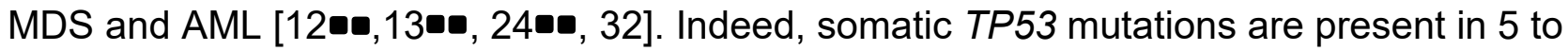
$10 \%$ of MDS cases and in $30 \%$ of secondary MDS patients arising after exposure to radiation or chemotherapy [20-21]. TP53 mutations in CHIP are associated with prior exposure to chemotherapy or radiotherapy $[12 \cdot \boldsymbol{\omega}, 13 \boldsymbol{\bullet}]$. Chemotherapy treatment resulted in expansion of both $p 53^{+/-}$and $p 53^{R 28 W /+}$ HSCs [13m- 24m, 32]. p53-/- HSCs are resistant to radiation [16] and we observed that TP53 mutations identified in CHIP confer radiation resistance, leading to selective expansion of TP53-mutant HSPCs (S.C. and Y.L., unpublished data). TP53 mutations in therapy-related CHIP are also associated with smoking [1200], suggesting that smoking-induced DNA damage may promote the expansion of hematopoietic clones with TP53 mutations.

Mutations in protein phosphatase $\mathrm{Mg}^{+} / \mathrm{Mn}^{+}$1D (PPM1D) have been identified in CHIP and myeloid neoplasms, with a enrichment in patients previously exposed to chemotherapy $[5-7,12 \boldsymbol{\bullet}, 13 \boldsymbol{\bullet}, 33 \boldsymbol{\bullet}, 34 \boldsymbol{\bullet}]$. PPM1D negatively regulates p53 and several proteins involved in the DNA damage response [35-36]. PPM1D mutations confer chemotherapy resistance, resulting in the selective expansion of PPM1D-mutant hematopoietic cells $[33 \bullet \mathbf{a}, 34 \boldsymbol{\bullet}]$. However, PPM1D mutants lack an advantage under bone marrow transplantation stress $[13 \boldsymbol{\bullet}, 33 \boldsymbol{- 1}, 34 \boldsymbol{\bullet}]$. Thus, mutant p53 appears to play distinct roles in driving clonal hematopoiesis compared to PPM1D mutants. 


\section{p53 in the pathogenesis of hematological malignancies}

The frequency of TP53 mutations in AML is approximately $10 \%$ [22]. However, in AML with complex karyotype, the frequency of p53 mutations and/or deletions is almost 70\% [23]. p53 mutations and/or deletions were thought to be secondary events that occur during leukemic transformation [37-39]. However, an aged individual with TP53 mutation in hematopoietic cells developed AML after acquiring additional chromosomal changes [5]. Interestingly, LiFraumeni syndrome (LFS), a rare, autosomal dominant disease, predisposes individuals to hereditary cancer linked to TP53 germline mutations, and some LFS patients develop MDS and AML as they age $[15,40]$, suggesting that TP53 mutations may be early events in leukemia development.

Most homozygous p53 knockout and mutant mice develop spontaneous tumors, including lymphoma and sarcoma, and die within 3 to 6 months after birth [30]. Since MDS is an age-related hematological disorder [20-21], we reasoned that heterozygous p53 mutant mice may develop myeloid malignancies with age. We maintained $p 53^{+/+}$and $p 53^{R 248 \mathrm{~W} /+}$ mice for more than a year and monitored tumor development. We found that some $p 53^{R 248 \mathrm{~W} /+}$ mice developed MDS with age based upon pathological analysis of BM and peripheral blood. Other $p 53^{R 248 W /+}$ mice developed lymphoma and sarcoma (S.C. and Y.L., unpublished data). Thus, we demonstrated that mutant p53 drives MDS in mice with age.

While coexisting mutations with TP53 mutations in AML are limited, previous studies indicate that TP53 mutations do co-occur with AML driver mutations in oncogenic signaling molecules such as NRAS and FMS-like tyrosine kinase receptor-3 (FLT3) [410๘, 42, 43-0]. Loss of p53 has been shown to cooperate with Nras mutations in AML development in mice.

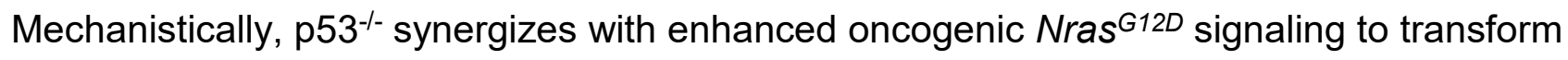
megakaryocyte-erythroid progenitors (MEPs) into leukemia-initiating cells (LICs) thereby 
driving AML development [43-a]. We found that mutant p53 cooperates with FLT3-ITD in chronic myeloid leukemia (CML) development in mice. Further, we found that mutant p53 enhanced the self-renewal potential of FLT3-ITD+ leukemia-initiating cells [44- $]$ ]. Given that TP53 mutations are present in both chronic and blast crisis phases of CML [45], our studies underscore the importance of mutant p53 in CML pathogenesis.

\section{Targeting mutant p53 to prevent CHIP progression and treat hematological malignancies}

TP53 is the most frequently mutated gene across all cancer types [14-15, 46-a]. The presence of mutant p53 predisposes to tumor development and is associated with ineffective therapeutic responses and unfavorable prognosis [15, 46-a]. Indeed, somatic TP53 mutations are associated with advanced disease and poor prognosis in both MDS and AML [19-23]. Despite these effects, no drug that abrogates the oncogenic functions of mutant p53 has yet been approved for the treatment of cancer [46-a]. To date, there are no effective treatment for MDS and AML patients with TP53 mutations, and most patients die within two years of diagnosis [19-23]. Thus, there is an urgent need to develop therapeutic strategies that can target mutant p53 and related pathways, enhancing our abilities to prevent CHIP progression and treat age-related diseases. While hematopoietic cell transplantation has curative potential, TP53 mutations are unfavorable prognostic markers for transplantation in MDS patients and negatively affect post-transplant survival [47an, 48]. While MDS and AML patients with TP53 mutations have been observed to have favorable clinical response and robust mutation clearance after receiving a 10-day courses of decitabine, the response is not durable [49]. Epigenetic factor EZH2 is rarely mutated in CHIP [5-7] and we found that genetic and pharmacological inhibition of EZH2 decrease the repopulating potential of p53 
mutant HSCs (S.C. and Y.L., unpublished data), suggesting that EZH2 may be a novel target for preventing CHIP progression in aged individuals with TP53 mutations.

\section{CONCLUSION}

Tumor suppressor gene TP53 was frequently mutated in individuals with CHIP and the frequency of TP53 mutations increases with age [5-9]. Recent findings suggest that several cellular stressors, including hematopoietic transplantation, genotoxic stress, and inflammation, promote the expansion of HSCs with TP53 mutations $[11 \cdot, 12 \boldsymbol{\bullet}, 13 \bullet \bullet, 24 \bullet \boldsymbol{\bullet}$, 310 ,32]. Further, TP53 mutations identified in CHIP cooperate with genetic and/or

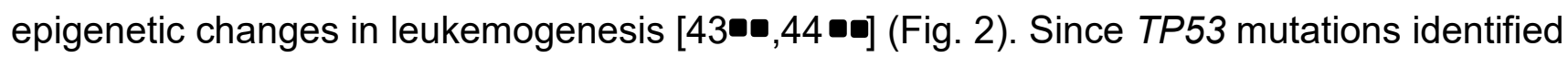
in CHIP are associated with increased risks of de novo and therapy-related hematological neoplasms $[5-9,12 \cdot \mathbf{m}, 13 \mathbf{m}]$, targeting mutant p53 and related pathways holds great potential in preventing CHIP progression and treating hematological malignancies.

\section{Acknowledgements}

None.

\section{Financial support and sponsorship}

This work was supported by the Office of the Assistant Secretary of Defense for Health Affairs, through the Bone Marrow Failure Research Program - Idea Development Award under Award No. W81XWH-18-1-0265 to YL. Opinions, interpretations, conclusions and recommendations are those of the author and are not necessarily endorsed by the Department of Defense. This work was also supported in part by NIH grants R56DK11952401 and R56AG05250 as well as a DoD Career Development Award W81XWH-13-1-0187 to YL. 


\section{Conflicts of interest}

There are no conflicts of interest. 


\section{REFERENCES AND RECOMMENDED READING}

Papers of particular interest, published within the annual period of review, have been

highlighted as:

- of special interest

- of outstanding interest

1. Jan M, Ebert BL, Jaiswal S. Clonal hematopoiesis. Semin Hematol 2017; 54: 43-50.

2. Sperling AS, Gibson CJ, Ebert BL. The genetics of myelodysplastic syndrome: from

-. clonal haematopoiesis to secondary leukaemia. Nat Rev Cancer 2017; 17:5-19.

Comprehensive review on CHIP development and MDS pathogenesis.

3. Steensma DP, Bejar R, Jaiswal S, et al. Clonal hematopoiesis of indeterminate potential and its distinction from myelodysplastic syndromes. Blood 2015;126:9-16.

4. Busque L, Buscarlet M, Mollica L, Levine RL. Concise Review: Age-Related Clonal

- Hematopoiesis: Stem Cells Tempting the Devil. Stem Cells 2018;36:1287-1294.

Comprehensive review on $\mathrm{ARCH}$.

5. Genovese G, Kähler AK, Handsaker RE, et al. Clonal hematopoiesis and blood-cancer risk inferred from blood DNA sequence. N Engl J Med 2014; 371: 2477-87.

6. Jaiswal S, Fontanillas P, Flannick J, et al. Age-related clonal hematopoiesis associated with adverse outcomes. N Engl J Med 2014;371: 2488-98.

7. Xie M, Lu C, Wang J, McLellan MD, et al. Age-related mutations associated with clonal hematopoietic expansion and malignancies. Nat Med 2014; 20: 1472-8. 
8. Young AL, Challen GA, Birmann BM, Druley TE. Clonal haematopoiesis harbouring AML-associated mutations is ubiquitous in healthy adults. Nat Commun 2016; $7: 12484$.

9. Zink F, Stacey SN, Norddahl GL, et al. Clonal hematopoiesis, with and without candidate driver mutations, is common in the elderly. Blood 2017;130:742-752.

10. Jaiswal S, Natarajan P, Silver AJ, et al. Clonal Hematopoiesis and Risk of Atherosclerotic Cardiovascular Disease. N Engl J Med 2017; 13:111-121.

11. Fuster JJ, MacLauchlan S, Zuriaga MA, et al. Clonal hematopoiesis associated

- with TET2 deficiency accelerates atherosclerosis development in mice. Science 2017; 355: 842-847.

Identifying a novel mechanism by which TET2 mutations drive cardiovascular diseases during aging.

12. Coombs CC, Zehir A, Devlin SM, et al. Therapy-Related Clonal Hematopoiesis in - Patients with Non-hematologic Cancers Is Common and Associated with Adverse Clinical Outcomes. Cell Stem Cell 2017; 21:374-382.e4.

Comprehensive clinical reports on therapy-related CHIP.

13.Wong TN, Miller CA, Jotte MRM, et al. Cellular stressors contribute to the expansion of - hematopoietic clones of varying leukemic potential. Nat Commun 2018; 9: 455. Showing distinct effects of hematopoietic transplantation and chemotherapy on the expansion of hematopoietic cells with different mutations. 
14. Levine AJ, Oren M. The first 30 years of p53: growing ever more complex. Nat Rev Cancer 2009; 9: 749-58.

15. Brosh R, Rotter V. When mutants gain new powers: news from the mutant p53 field. Nat Rev Cancer 2009;9: 701-13.

16. Liu Y, Elf SE, Miyata Y, et al. p53 Regulates Hematopoietic Stem Cell Quiescence. Cell Stem Cell 2009; 4: 37-48.

17. Asai T, Liu Y, Di Giandomenico S, et al. Necdin, a p53 target gene, regulates the quiescence and response to genotoxic stress of hematopoietic stem/progenitor cells. Blood 2012; 120: 1601-12.

18. Kenzelmann Broz D, Spano Mello S, Bieging KT, et al. Global genomic profiling reveals an extensive p53-regulated autophagy program contributing to key p53 responses. Genes Dev 2013; 27: 1016-31.

19. Peller S, Rotter V. TP53 in hematological cancer: low incidence of mutations with significant clinical relevance. Hum Mutat 2003; 21: 277-84.

20. Lindsley RC, Ebert BL. Molecular pathophysiology of myelodysplastic syndromes. Annu Rev Pathol 2013; 8: 21-47.

21. Bejar R, Stevenson K, Abdel-Wahab O, et al. Clinical effect of point mutations in myelodysplastic syndromes. N Engl J Med 2011;364: 2496-506.

22. Grossmann V, Schnittger S, Kohlmann A, et al. A novel hierarchical prognostic model of AML solely based on molecular mutations. Blood 2012; 120: 2963-72. 
23. Rücker FG, Schlenk RF, Bullinger L, et al. TP53 alterations in acute myeloid leukemia with complex karyotype correlate with specific copy number alterations, monosomal karyotype, and dismal outcome. Blood 2012; 119: 2114-21.

24. Chen S, Gao R, Yao C, et al. Genotoxic stresses promotes the clonal expansion of - hematopoietic stem cells expressing mutant p53. Leukemia 2018; 32:850-854.

Showing the effects of genotoxic stresses, including chemotherapy and radiation, on clonal expansion of hematopoietic progenitor cells with TP53 mutations identified in CHIP. 25. Beerman I, Rossi DJ. Epigenetic Control of Stem Cell Potential during Homeostasis, Aging, and Disease. Cell Stem Cell 2015; 16: 613-25.

26. Sun D, Luo M, Jeong M, et al. Epigenomic profiling of young and aged HSCs reveals concerted changes during aging that reinforce self-renewal. Cell Stem Cell 2014; 14:673-88.

27.Zhu J, Sammons MA, Donahue G, et al. Gain-of-function p53 mutants co-opt chromatin pathways to drive cancer growth. Nature 2015; 525: 206-11.

28. Bracken AP, Helin K. Polycomb group proteins: navigators of lineage pathways led astray in cancer. Nat Rev Cancer 2009; 9: 773-84.

29. Mochizuki-Kashio M, Mishima Y, Miyagi S, et al. Dependency on the polycomb gene Ezh2 distinguishes fetal from adult hematopoietic stem cells. Blood 2012;118: 655361.

30. Song $\mathrm{H}$, Hollstein $\mathrm{M}, \mathrm{Xu}$ Y. p53 gain-of-function cancer mutants induce genetic 
instability by inactivating ATM. Nat Cell Biol 2007; 15: 376-88.

31. Cai Z, Kotzin JJ, Ramdas B, et al. Inhibition of Inflammatory Signaling in Tet2 Mutant

- Preleukemic Cells Mitigates Stress-Induced Abnormalities and Clonal Hematopoiesis.

Cell Stem Cell 2018; 23:833-849.e5.

Identifying a novel mechanism by which TET2 mutations drive clonal hematopoiesis.

32. Wong TN, Ramsingh G, Young AL, et al. Role of TP53 mutations in the origin and evolution of therapy-related acute myeloid leukaemia. Nature 2015;518: 552-5.

33. Hsu JI, Dayaram T, Tovy A, et al. PPM1D Mutations Drive Clonal Hematopoiesis in

- Response to Cytotoxic Chemotherapy. Cell Stem Cell 2018; 23: 700-713.e6.

Determining the role of PPM1D mutations in clonal hematopoiesis.

34. Kahn JD, Miller PG, Silver AJ, et al. PPM1D-truncating mutations confer resistance to

- chemotherapy and sensitivity to PPM1D inhibition in hematopoietic cells. Blood 2018; 132:1095-1105.

Determining the role of PPM1D mutations in clonal hematopoiesis.

35. Fiscella M, Zhang H, Fan S, et al. Wip1, a novel human protein phosphatase that is induced in response to ionizing radiation in a p53-dependent manner. Proc Natl Acad Sci USA 1997; 94:6048-6053.

36. Lu X, Nguyen TA, Moon SH, et al. The type 2C phosphatase Wip1: an oncogenic regulator of tumor suppressor and DNA damage response pathways. Cancer Metastasis Rev 2008; 27:123-135. 
37. Nakai H, Misawa S, Toguchida J, et al. Frequent p53 gene mutations in blast crisis of chronic myelogenous leukemia, especially in myeloid crisis harboring loss of a chromosome 17p. Cancer Res 1992; 52:6588-93.

38. Harutyunyan A, Klampfl T, Cazzola M, et al. p53 lesions in leukemic transformation. $\mathrm{N}$ Engl J Med 2011; 364:488-90.

39. Rampal R, Ahn J, Abdel-Wahab O, et al. Genomic and functional analysis of leukemic transformation of myeloproliferative neoplasms. Proc Natl Acad Sci U S A 2014; 111:E5401-10.

40. Talwalkar SS, Yin CC, Naeem RC, et al. Myelodysplastic syndromes arising in patients with germline TP53 mutation and Li-Fraumeni syndrome. Arch Pathol Lab Med 2010; 134:1010-5.

41. Prokocimer M, Molchadsky A, Rotter V. Dysfunctional diversity of p53 proteins in adult

- acute myeloid leukemia: projections on diagnostic workup and therapy. Blood 2017;130:699-712.

Comprehensive review of the role of mutant p53 in pathogenesis and treatment of AML.

42. Garg M, Nagata Y, Kanojia D, Mayakonda A, Yoshida K, Haridas Keloth S et al. Profiling of somatic mutations in acute myeloid leukemia with FLT3-ITD at diagnosis and relapse. Blood 2015;126:2491-501.

43. Zhang J, Kong G, Rajagopalan A, et al. p53-/- synergizes with enhanced NrasG12D

- signaling to transform megakaryocyte-erythroid progenitors in acute myeloid leukemia. Blood 2017;129:358-370.

Showing the synergy between p53 loss and NRAS mutation in AML pathogenesis in mice. 44. Nabinger S, Chen S, Yao C, et al. Mutant p53 enhances leukemia-initiating cell self-

- renewal to promote leukemia development. Leukemia. 2019 Jan 23. doi: 
10.1038/s41375-019-0377-0. [Epub ahead of print]

Showing the synergy between mutant p53 and FLT3 mutation in CML pathogenesis in mice.

45. Menezes J, Salgado RN, Acquadro F, et al. ASXL1, TP53 and IKZF3 mutations are present in the chronic phase and blast crisis of chronic myeloid leukemia. Blood Cancer J 2013;3:e157.

46. Sabapathy K, Lane DP. Therapeutic targeting of p53: all mutants are equal, but some mutants are more equal than others. Nat Rev Clin Oncol 2018;15:13-30.

Comprehensive review on therapeutic targeting of mutant p53 in human cancer. 47. Lindsley RC, Saber W, Mar BG, et al. Prognostic Mutations in Myelodysplastic - Syndrome after Stem-Cell Transplantation. N Engl J Med 2017; 376:536-547. Showing that TP53 mutations are poor prognostic markers in MDS after stem cell transplantation.

48. Yoshizato T, Nannya Y, Atsuta Y, et al.. Genetic abnormalities in myelodysplasia and secondary acute myeloid leukemia: impact on outcome of stem cell transplantation. Blood 2017;129:2347-2358.

49. Welch JS, Petti AA, Miller CA, et al. TP53 and Decitabine in Acute Myeloid Leukemia and Myelodysplastic Syndromes. N Engl J Med 2016; 375: 2023-2036. 


\section{FIGURE LEGENDS}

Figure 1. Tumor suppressor gene TP53 ranks in the top five among genes that were mutated in clonal hematopoiesis of indeterminate potential (CHIP).

Figure 2. TP53 mutations identified in CHIP utilize distinct mechanisms to promote HSC expansion during aging. Expanded mutant HSCs become fully transformed leukemiainitiating cells (LICs) after acquiring additional genetic and/or epigenetic changes. 
Figure 1

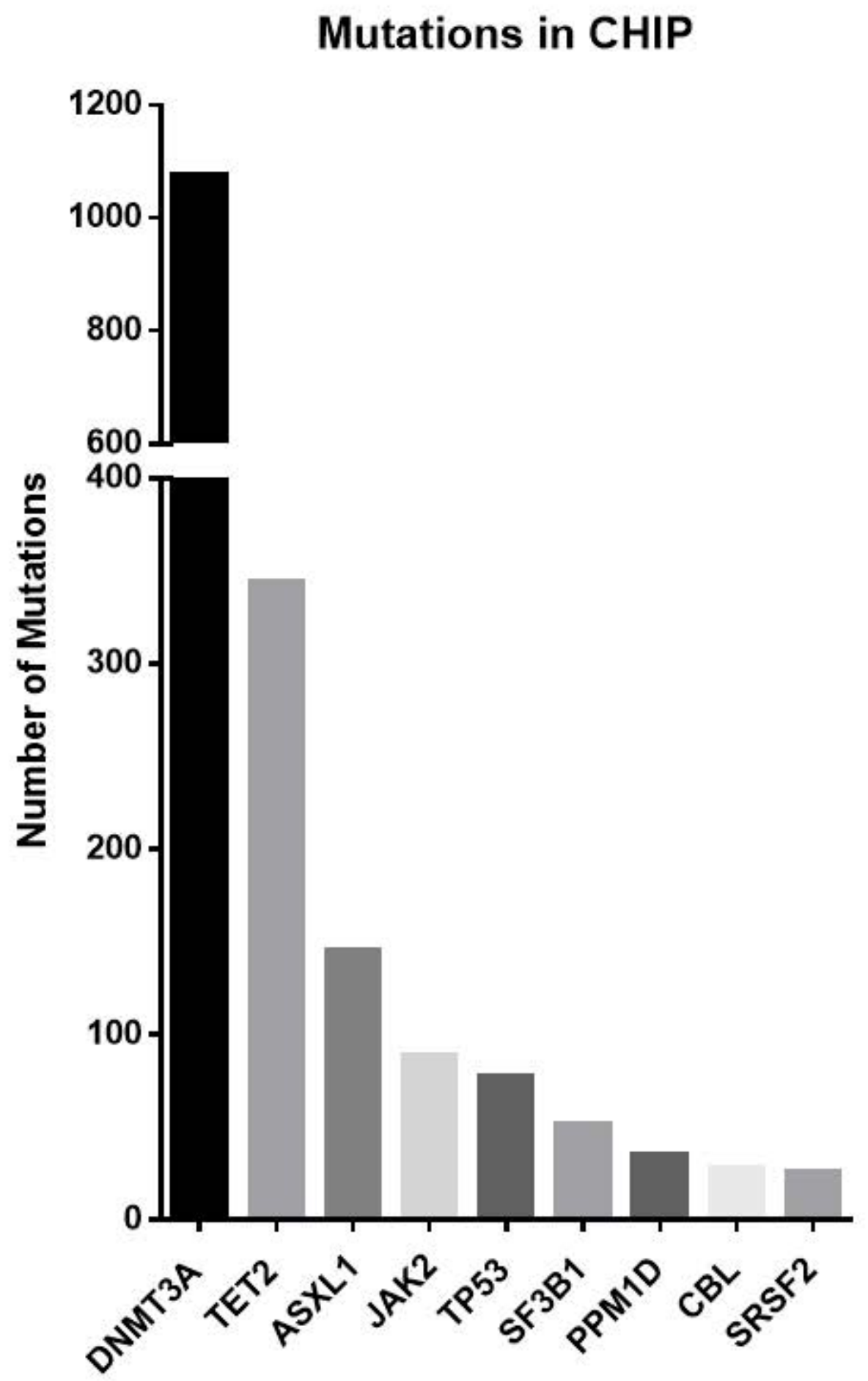




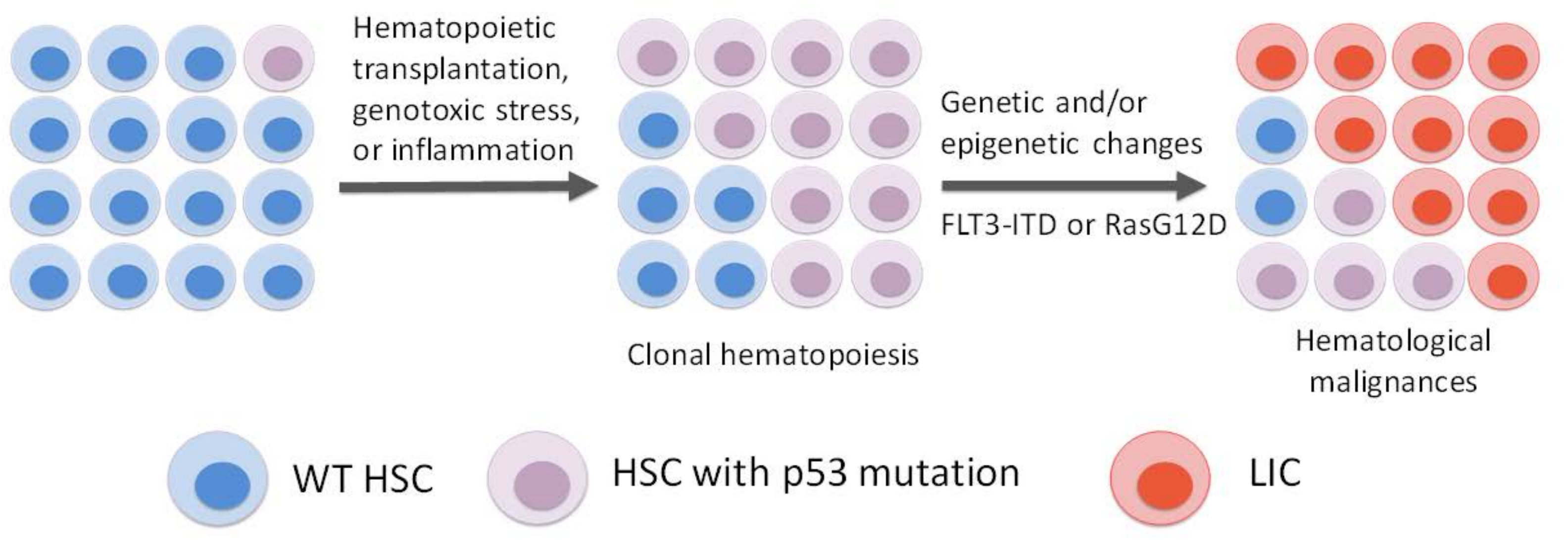

